$\S=-1$

\title{
Nanographene Sheet Immobilized Transition Metal Complexes for C-C Coupling Reactions
}

\author{
Saroja Anuma ${ }^{1}$, Badekai Ramachandra Bhat ${ }^{2 *}$ \\ ${ }^{1}$ Catalysis and Material Chemistry Laboratory, National Institute of Technology Karnataka, Mangalore, Karnataka, India-575025 \\ ${ }^{2}$ Department of Chemistry, National Institute of Technology Karnataka, Mangalore, Karntaka, India-575025 \\ *Corresponding author E-mail: ram@nitk.edu.in
}

\begin{abstract}
Transition metal Schiff base complexes covalently immobilized on the surface of amino functionalized graphene sheet to catalyze C-C coupling reaction is reported. Graphene sheet which synthesized using mechanical exfoliation and then by differential oxidative reduction phenomenon of graphite. The schiff base ligand is prepared using aldehyde and amino groups containing compounds which further treated with metal salts to form metal complexes. The as-synthesized metal complexes was then incorporated into amino functionalized nanosheets which are characterized using different spectrochemical techniques. The catalytic investigation of the asprepared catalyst were done by the cross coupling of aryl halides and aryl boronic acids to form biaryls as product in C-C cross coupling reaction. The yield of the products were high and selective which were analyzed using Gas chromatography technique. The advantage of this catalyst is high stability, recyclability easy recovery of catalyst, environmentally benign and mild reaction condition.
\end{abstract}

Keywords: Biaryl; Schiff Base; metal complexes; graphene sheet

\section{Introduction}

The synthesize of biaryl compounds is of great interest due to its broad area of its applications in natural products ${ }^{1}$, pharmaceuti$\mathrm{cals}^{2}$, agrochemicals ${ }^{3}$ and functionalized polymer materials ${ }^{4}$. Since its discovery, the cross-coupling involving organoboron reagent and organic halides or pseudo halides is one among the important methodology for the construction of carbon-carbon bonds and a viable protocol in organic chemistry because of the stability of the starting materials, tolerance towards various functional groups and compatibility to a wide range of solvents. With the invent of various transition metal complexes in coordination chemistry it paved a way for the constructing the symmetrical biaryl backbone and also the rate of reactions have increased manifold ${ }^{5}$. Although a variety of metallic reagents have been applied in the coupling reaction earlier, a promising approach in the development of a ligand framework which accommodate the modification during catalysis is highly desirable ${ }^{6}$.

Different strategies have been attempted/developed for imparting the high activity of the homogeneous catalysts along with stability ${ }^{7}$. But the greater disadvantage found in the critical area of catalytic study of coupling reaction during the reaction pathway is that the inhibition and deactivation of the catalyst i.e., due to the homogeneous catalysts which are composed of transition metal complexes. An alternative approach is the heterogenization of the transition metal complexes which is an interesting approach, can offer high reaction rate, selectivity and facile recovery ${ }^{8,9}$. Such unique materials with tailored composition (hybrid organic-inorganic core shell nanostructure became building blocks for increasing the efficacy in most of the metal catalyzed coupling reactions ${ }^{10-12}$. In order to explore and compare the catalytic performance, there are some key aspects such as surface area, size distributions, surface functionality and structure have to be particularly studied and the metal-support interaction plays a pivotal role for the activity rationale ${ }^{13}$.

Despite of all the comprehensive strategies, in order to yield a maximum product with aryl halides to form carbon-carbon bond, with the improvement of transition metal complexes onto different supports, a viable approach is heterogenization of various carbon nanomaterials such as carbon nanotube, carbon nanofibers, carbon nanospheres and fullerene. Graphene, a novel member to carbon material family having $2 \mathrm{D}$ sheets of $\mathrm{sp}^{2}$ hybridized carbon atoms attracted interest in the field of catalysis and energy storage due to its electronic conductivity, mechanical stiffness, large surface area and excellent chemical stability ${ }^{14-16}$.

Nanographene sheets having abundant functional groups such as carboxyl, carbonyl, hydroxyl and epoxide etc., on its basal planes as well as edges makes it less expensive compared to mesoporous zeolites such as MCM-41 and SBA-45. Because of its unique nanostructure, it has various applications such as polymer composites, sensors and catalysis. Hybrid materials comprising both inorganic Nanographene and organo amine groups facilitates the strong binding to the metal atom which enhances the catalytic performance.

The aim for our work is to improve the yield for aryl halides and recyclability of the improved catalytic system using metal immobilized Nanographene sheet. The structure and morphology of the as-prepared metal immobilized Nanographene sheet were characterized by Fourier Transform Infrared Spectroscopy (FTIR) and Scanning electron microscope (SEM). The mechanism of the C-C coupling reaction using nanocatalysts was also discussed. 


\section{Materials and Method}

\subsection{General}

Graphite, amino functionalization group, metal salts, arylboronic acids and aryl halides were purchased from sigma-Aldrich. Ethanol, toluene, acetonitrile, DMSO, hydrogen peroxide, sulphuric acid, phosphoric acid are obtained from Finar chemicals limited. Caesium carbonate, potassium carbonate, sodium hydroxide, potassium hydroxide, sodium nitrate, sodium carbonate and potassium permanganate are obtained from lobo chemie limited.

\subsection{Synthesis of Metal Immobilized Amino Functional- ized Nanographene Sheets}

Nanographene sheet was prepared using $1.5 \mathrm{~g}$ of graphite and $2.5 \mathrm{~g}$ of $\mathrm{NaNO}_{3}$ were mixed with $108 \mathrm{ml}$ of $\mathrm{H}_{2} \mathrm{SO}_{4}$ and $12 \mathrm{ml}$ of $\mathrm{H}_{3} \mathrm{PO}_{4}$ under rigorous stirring in an ice bath. Later, $15 \mathrm{~g}$ of $\mathrm{KMnO}_{4}$ added and the temperature of the mixture maintained at $5{ }^{\circ} \mathrm{C}$. The suspension stirred for $60 \mathrm{~min}$. Deionized water was further added and $15 \mathrm{ml}$ of $\mathrm{H}_{2} \mathrm{O}_{2}$ was added after $5 \mathrm{~min}$. The reaction product was centrifuged, washed with deionized water and dried in oven. The amino functionalized groups stirred along with graphene sheets in absolute ethanol under reflux conditions for 24 hand filtered it. The metal complexes refluxed with amino functionalized graphene sheets under reflux conditions in ethanol for $24 \mathrm{~h}$ and filtered it.

\section{Result and Discussion}

In this work, the metal immobilized Nanographene sheet catalys shows improved reactivity due to the covalent interaction of amino functionalized Nanographene sheet with transition metal complex. Herein, we reported a series of physicochemical characterizations such as FTIR and SEM analysis were conducted on the catalyst to understand the structure and composition of the catalyst. The infrared spectra were recorded in $\mathrm{KBr}$ disks using a BrukerAlpha ECO-ATR FTIR in the range $500-4000 \mathrm{~cm}^{-1}$. SEM was recorded on Scanning Electron Microscopy (SEM) JSM-6380LA.

\subsection{FTIR (Fourier transform Infrared Spectroscopy)}

The FT-IR spectra of the pure Nanographene sheet, amino functionalized Nanographene sheet and metal immobilized Nanographene sheet. For pure Nanographene sheet, the strong band at $1725,3390,1058,1220$ and $1621 \mathrm{~cm}^{-1}$ represents the carboxylic acid, hydroxyl group, epoxy $(\mathrm{C}-\mathrm{O})$, phenol and $\mathrm{C}=\mathrm{C}$ in their scaffold $^{17}$. The FT-IR spectrum of amino functionalized Nanographene sheet have the band at $3420.2,1620 \mathrm{~cm}^{-1}$ were attributed to $\mathrm{N}-\mathrm{H}$ and C-N vibrations. The new bands at $685 \mathrm{~cm}^{-1}$ was attributed to the stretching of metal- $\mathrm{N}$ bond and confirmed the coordination of metal with Nanographene sheet.

\subsection{SEM (Scanning Electron Spectroscopy)}

The surface morphology and microstructural features of Nanographene sheet and metal immobilized Nanographene sheet were analyzed using SEM. The 2D structure of Nanographene sheet and composite metal immobilized Nanographene sheet showed twisted Nanosheets in disordered phase and most of the wrinkles with crumpling features on their surface. Due to a lot of folded edges and protrusions which enables to form metal immobilized onto Nanographene sheet. These sites may serve as idealistic templates which help in reactant molecules to form their target transformation of Nanographene sheet.

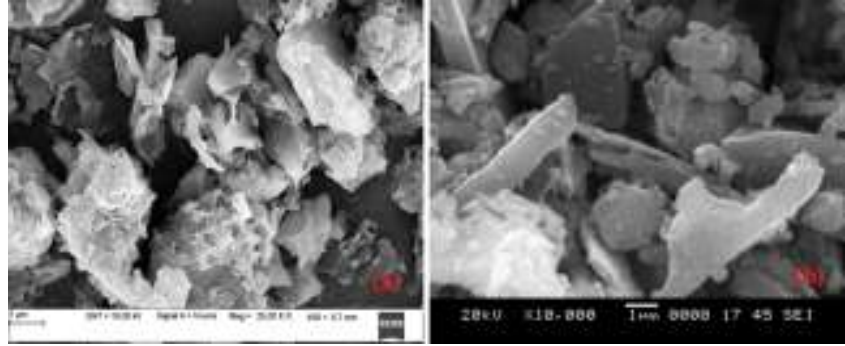

Figure 1: The SEM morphology of a) GO and b) Synthesized catalyst

\subsection{C-C Coupling Reaction}

Aryl halide $(1 \mathrm{mmol})$ and phenyl boronic acid $(1.5 \mathrm{mmol})$ were added to a flask containing the metal immobilized Nanographene sheet $(0.5 \mathrm{~mol} \%)$ and $\mathrm{K}_{2} \mathrm{CO}_{3}(3 \mathrm{mmol})$ in ethyl alcohol $(3.5 \mathrm{mmol})$ under reflux conditions up to $12 \mathrm{~h}$. Upon the completion of the reaction (monitored using thin layer chromatography), the reaction mixture was cooled to room temperature. At the stated time, samples were taken from the reaction mixture and analyzed using Gas Chromatography (GC) (Shimadzu 2014, Japan), siloxane Restek capillary column (30m length and $0.25 \mathrm{~mm}$ diameter) and Flame Ionization Detector (FID). The catalytic activity of the synthesized catalyst were carried the model reaction. In general the application of this catalyst, the coupling reactions of various substituted aryl halides and phenyl boronic acids were carried out in the presence of base $\mathrm{K}_{2} \mathrm{CO}_{3}$ using EtOH as an ecofriendly solvent. The product yield was good for the reaction of bromobenzene with phenylboronic acid (Table 1). The results were observed that the reaction could be completed in $12-24 \mathrm{~h}$ with moderate to good yields, depending upon the type of substrates having either an electron donating or electron withdrawing group.

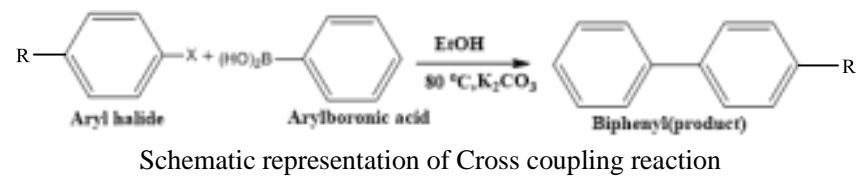

Table 1: The catalytic activity of $\mathrm{C}-\mathrm{C}$ coupling reactions using different aryl bromides, Calculated by GC analysis, aryl bromides (1.0mmol), arylboronic acid $(1.5 \mathrm{mmol})$, solvent $(3.5 \mathrm{mmol})$, catalyst $(0.5 \mathrm{~mol} \%)$ and $\mathrm{K}_{2} \mathrm{CO}_{3}(3.0 \mathrm{mmol})$.

\begin{tabular}{|c|c|c|c|}
\hline Entry & $\mathbf{X}$ & $\mathbf{R}$ & Yield(\%) \\
\hline 1 & $\mathrm{Br}$ & $\mathrm{COCH}_{3}$ & 78.00 \\
\hline 2 & $\mathrm{Br}$ & $\mathrm{CH}_{3}$ & 60.00 \\
\hline 3 & $\mathrm{Br}$ & $\mathrm{NHCOCH}_{3}$ & 74.50 \\
\hline 4 & $\mathrm{Br}$ & $\mathrm{H}$ & 90.00 \\
\hline 5 & $\mathrm{Br}$ & $\mathrm{OCH}_{3}$ & 64.22 \\
\hline 6 & $\mathrm{Br}$ & $\mathrm{CN}$ & 88.00 \\
\hline
\end{tabular}

\subsubsection{Effect of Solvent:}

The catalytic activity of the synthesized catalysts was studied using $\mathrm{C}-\mathrm{C}$ coupling reaction of phenyl bromide and phenylboronic acid (Base: $\mathrm{K}_{2} \mathrm{CO}_{3}$, Concentration of catalyst: $0.5 \mathrm{~mol} \%$ ) under reflux conditions in various solvents. Among all the solvents used (Figure 2), the highest catalytic performance was observed with Ethanol and is chosen as the optimum solvent for carrying out the reactions. 


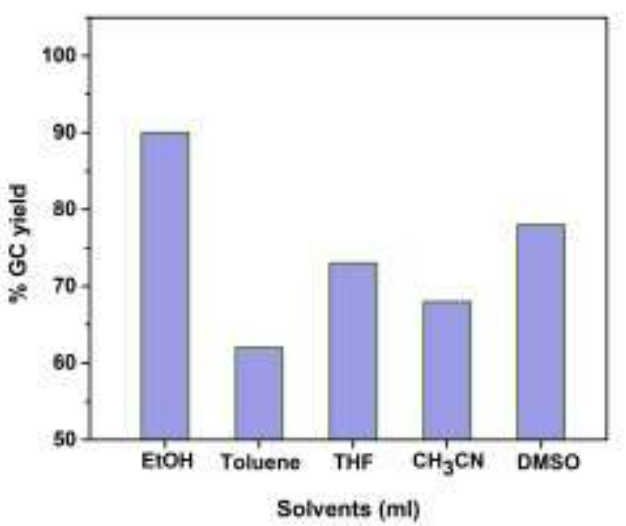

Fig. 2: The effect of solvent on the rate of the reaction

\subsubsection{Effect of Catalyst Amount}

The catalysts loading in a reaction significantly monitors the catalyst efficiency in the coupling reaction and hence the amount of catalyst required is one of the parameters to be studied for better conversion. The yield was found to increase with the increase in the amount of catalyst used in (Figure 3). However, after the catalysts amount of $0.5 \mathrm{~mol} \%$, the reaction yield remains constant and there is no significant change in the rate of reaction due to the deactivation of the catalysts.

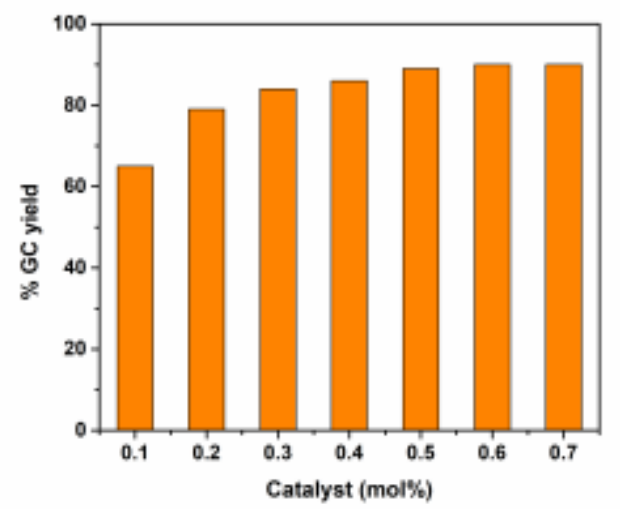

Fig. 3: The effect of catalyst amount with regards to GC yield

\subsubsection{Effect of Time and Base}

Moreover, the product formation and its yield depends on reaction time as well as the amount of base. This was investigated by analyzing the reaction mixture at regular interval of time and the base effect (Figure $4 a, b$ ). The rate of product formation observed to increase till $12 \mathrm{~h}$ and beyond that even increase in time period, there is no probable change in extent of product formation almost remains significantly constant. The ideal time for optimum reaction conditions is $12 \mathrm{~h}$. An internal standard was used and it is calibrated against each one of the coupling product. The ideal base is $\mathrm{K}_{2} \mathrm{CO}_{3}$ at the optimized conditions for the reaction for obtaining the maximum product during the coupling reaction.
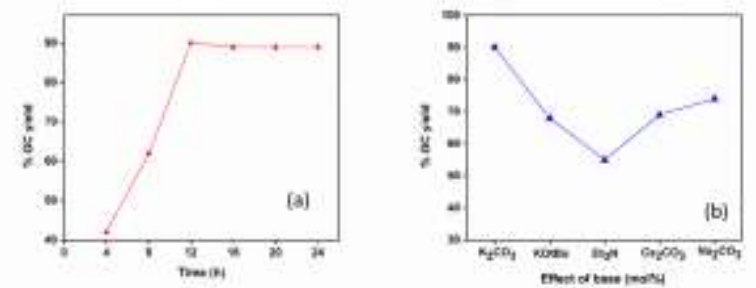

Fig. 4: a) the effect of time during reaction and b) the effect of different bases in the reaction.

\subsubsection{Recyclability Studies}

Recyclability of the catalyst was done to observe the recycling of the heterogeneous catalyst which help in practical applications. The catalyst were effective for 4 consecutive cycles and the product yield decreased gradually.

\section{Conclusion}

In conclusion, we developed a methodology for the metal immobilized Nanographene sheets through a surface modification. The metal immobilized Nanographene sheets complex catalyst was found to be a highly effective and recyclable catalyst for the synthesis of biphenyl as products from the $\mathrm{C}-\mathrm{C}$ coupling reaction of substituted aryl halides and phenylboronic acid. Heterogeneous nanocatalysts is usually active, possesses high surface area, and its thermal and chemical stabilities were confirmed using various characterization technique through spectroscopic methods and surface analysis. This recoverable and reusable catalyst is benign, low cost and be handled easily. Indepth analysis in heterogeneous catalysis were done in future studies in various organic reactions were expected.

\section{Acknowledgement}

The authors thank the, National Institute of Technology Karnataka (NITK), Surathkal for providing fellowship and research facilities.

\section{References}

[1] A. Suzuki, (1999) "Recent advances in the cross-coupling reactions of organoboron derivatives with organic electrophiles 1995-1998", Journal of organometallic chemistry, 576(1), 147-168.

[2] D. Zhang and Q. Wang, (2015) "Palladium catalyzed asymmetric Suzuki-Miyaura coupling reactions to axially chiral biaryl compounds: Chiral ligands and recent advances Coordination Chemistry Reviews", 286, 1-16.

[3] I. P. Beletskaya, A.V. Cheprakov, (2000) "The Heck Reaction as a Sharpening Stone of Palladium Catalysis Chemical Reviews", 100, 3009-3066.

[4] S. Kotha, K. Lahiri and D.Kashinath, (2002) "Recent applications of the Suzuki-Miyaura cross-coupling reaction in organic synthesis", Tetrahedron, 58, 9633-9695.

[5] C. Torborga and M. Beller, (2009) "Recent Applications of Palladium-Catalyzed Coupling Reactions in the Pharmaceutical, Agrochemical and Fine Chemical Industries", Advanced Synthesis and Catalysis, 351, 3027-3043.

[6] Aparna. I. Bhat, Fawad Ianam and B. Ramachandra Bhat, (2013) "Nickel catalyzed one pot synthesis of biaryls under air at room temperature", RSC Advances, 44(3) 22191- 22198.

[7] A. Corma and H. Garcia, (2008) "Crossing the Borders Between Homogeneous and Heterogeneous Catalysis: Developing Recoverable and Reusable Catalytic Systems", Topics in Catalysis, 48, 8-31.

[8] H. Lee, (2014) "Utilization of shape-controlled nanoparticles as catalysts with enhanced activity and selectivity RSC Advances", 4, 41017-41027.

[9] F. Zaera, (2013) "Nanostructured materials for applications in heterogeneous catalysis", Chemical Society Reviews 42, 2746-2762.

[10] A. Kim, J. C. Park, M. Kim, E. Heo, H. Song, K.H. Park, (2014) "Suzuki Coupling Reaction Using Hybrid Pd Nanoparticles", Journal of Nanoscience and Nanotechnology, 14, 1872-1883.

[11] W. Sheng, Q. Yang, and J, Weng, (2011) "Application of Noble Metal Nanoparticles in Organic Reactions", Current Organic Chemistry, 15, 3692-3705.

[12] L. Djakovitch and F. Felpin, (2014) "Direct C sp ${ }^{2}-\mathrm{H}$ and $\mathrm{C} \mathrm{sp}$-H Arylation Enabled by Heterogeneous Palladium Catalysts", ChemCatChem, 6, 2175-2187.

[13] Suellen D. T. de Barros, Jaqueline D. Senra, Elizabeth R. Lachter and Luiz Fernando B. Malta, (2016) "Metal-catalyzed crosscoupling reactions with supported nanoparticles: Recent developments and future directions", Catalysis reviews, 58, (4), 439-496. 
[14] Y. Zhao, C. G. Hu, Y.Hu, H. H. Cheng, G. Q. Shi and L. T. Qu, (2012) "A Versatile, Ultralight, Nitrogen-Doped Graphene Framework", Angewandte Chemie International Edition, 51, 1137111375 .

[15] C. H. Zhang, L. Fu, N. Liu, M. H. Liu, Y. Y. Wang and Z. F. Liu, (2011) "Synthesis of Nitrogen-Doped Graphene Using Embedded Carbon and Nitrogen Sources", Advanced Material Letters, 23(8), 1020-1024.

[16] Y. Wang, Y. Y. Shao, D. W. Matson, J. H. Li and Y.H. Lin, (2010) "Nitrogen-Doped Graphene and Its application in Electrochemical Biosensing", ACS Nano, 4, 1790-1798.

[17] Zhifang Li, Shujie Wu, (2013) "Immobilized $\mathrm{Cu}($ II) and $\mathrm{Co}(\mathrm{II})$ salen complexes on graphene oxide and their catalytic activity for aerobic epoxidation of styrene", New Journal of Chemistry, 37(5), 1561-1568. 